\title{
Prospective validation of right ventricular role in primary graft dysfunction after lung transplantation
}

\author{
Purificación Pérez-Terán (10 ${ }^{1,2}$, Oriol Roca $\mathbb{1}^{3,4}$, José Rodríguez-Palomares ${ }^{5}$, \\ Juan C. Ruiz-Rodríguez ${ }^{3}$, Ana Zapatero ${ }^{1}$, Joaquim Gea ${ }^{4,6,7}$, Joaquim Serra ${ }^{3}$, \\ Arturo Evangelista ${ }^{5}$ and Joan R. Masclans ${ }^{1,4,7}$
}

\begin{abstract}
Affiliations: ${ }^{~}$ Critical Care Dept, Hospital del Mar-Parc de Salut Mar, Institut Mar d'Investigacions mèdiques (IMIM), Barcelona, Spain. ${ }^{2}$ Departament de Medicina, Universitat Autònoma de Barcelona, Barcelona, Spain. ${ }^{3}$ Critical Care Dept, Vall d'Hebron University Hospital, Institut de Recerca Vall d'Hebron (VHIR), Barcelona, Spain. ${ }^{4}$ Ciber de Enfermedades Respiratorias, Instituto de Salud Carlos III, Madrid, Spain. ${ }^{5}$ Cardiology Dept, Vall d'Hebron University Hospital, VHIR, Barcelona, Spain. ${ }^{6}$ Respiratory Dept, Hospital del Mar-Parc de Salut Mar, IMIM, Barcelona, Spain. ${ }^{7}$ Universitat Pompeu Fabra, Barcelona, Spain.
\end{abstract}

Correspondence: Purificación Pérez-Terán, Critical Care Dept, Hospital del Mar-Parc de Salut Mar, Passeig Marítim, 25-29, 08003, Barcelona, Spain. E-mail: pperezQparcdesalutmar.cat

ABSTRACT Primary graft dysfunction is a significant cause of lung transplant morbidity and mortality, but its underlying mechanisms are not completely understood. The aims of the present study were: 1) to confirm that right ventricular function is a risk factor for severe primary graft dysfunction; and 2) to propose a clinical model for predicting the development of severe primary graft dysfunction.

A prospective cohort study was performed over 14 months. The primary outcome was development of primary graft dysfunction grade 3 . An echocardiogram was performed immediately before transplantation, measuring conventional and speckle-tracking parameters. Pulmonary artery catheter data were also measured. A classification and regression tree was made to identify prognostic models for the development of severe graft dysfunction.

70 lung transplant recipients were included. Patients who developed severe primary graft dysfunction had better right ventricular function, as estimated by cardiac index $\left(3.5 \pm 0.8\right.$ versus $2.6 \pm 0.7 \mathrm{~L} \cdot \mathrm{min}^{-1} \cdot \mathrm{m}^{-2}$, $\mathrm{p}<0.01)$ and basal longitudinal strain $(-25.7 \pm 7.3 \%$ versus $-19.5 \pm 6.6 \%, \mathrm{p}<0.01)$. Regression tree analysis provided an algorithm based on the combined use of three variables (basal longitudinal strain, pulmonary fibrosis disease and ischaemia time), allowing accurate preoperative discrimination of three distinct subgroups with low (11-20\%), intermediate (54\%) and high (75\%) risk of severe primary graft dysfunction (area under the receiver operating characteristic curve 0.81).

Better right ventricular function is a risk factor for the development of severe primary graft dysfunction. Preoperative estimation of right ventricular function could allow early identification of recipients at increased risk, who would benefit the most from careful perioperative management in order to limit pulmonary overflow.

@ERSpublications

RV function assessment before LT by STE is essential for identification of recipients with higher risk of PGD http://ow.ly/fidz3036kpG

This article has supplementary material available from erj.ersjournals.com

Received: Dec 172015 | Accepted after revision: Aug 052016 | First published online: Oct 062016

Support statement: P. Pérez-Terán is the recipient of a Rio Hortega grant (ref. CM12/00216) from the Instituto de Salud Carlos III, Ministerio de Ciencia e Innovación.

Conflict of interest: None declared.

Copyright OERS 2016 


\section{Introduction}

Although lung transplantation (LT) has become an increasingly common procedure in recent years, it has consistently lagged behind other organ transplants in terms of survival rates [1]. Primary graft dysfunction (PGD) is a severe form of acute respiratory distress syndrome induced fundamentally by ischaemiareperfusion injury $[2,3]$. Its prevalence varies between $12 \%$ and $55 \%$, depending on the time point chosen for grading $[1,4,5]$, and it remains a significant cause of post-transplantation morbidity and mortality $[1,4,6-8]$. Consequently, identification of the risk factors associated with the appearance of PGD is an issue of great clinical interest; it would broaden our understanding of PGD pathophysiology, and would thus aid the design of new treatments and the identification of high-risk patients likely to benefit the most from early specific interventions.

Several studies have aimed to identify the clinical risk factors associated with PGD development after LT [2, 4, 8-11]. Preoperative pulmonary hypertension $(\mathrm{PH})$ is one of the most frequently described $[4,8,9]$. However, the pathophysiological mechanisms that underlie this association are not fully understood. One hypothesis is that the right ventricle (RV) may be a key element in the development of PGD. However, although the importance of the RV and its interactions with pulmonary circulation were demonstrated for the first time four centuries ago, this area remains largely understudied.

$\mathrm{PH}$ is common among patients with respiratory diseases who undergo LT [12]. In these patients, right ventricular function (RVF) may vary from the normal to a compensated (hypertrophied) and, thereafter, a decompensated state [13-15]. An interesting hypothesis is that the haemodynamic forces of a "well-trained" hyperdynamic RV contracting against the reduced pulmonary vascular resistance of the implanted lungs may induce an exacerbation of ischaemia-reperfusion lung injury and may thus play a role in the development of PGD [16-18]. However, the quantification of RVF still remains a challenge because, due to its complex geometry, conventional two-dimensional (2D) echocardiography does not provide a comprehensive evaluation [19]. Speckle-tracking echocardiography (STE) has been proposed as an alternative approach for determining RVF, allowing the quantification of myocardial deformation from standard 2D echo-images without the problem of angle dependence [20]. In a previous retrospective study, our group identified RVF (measured by basal longitudinal strain (BLS)) as a possible independent risk factor for severe PGD development [5].

The main objective of the present study was to prospectively confirm that RVF is a risk factor for PGD. Secondarily, we also aimed to perform a prospective validation of BLS as a surrogate of invasive cardiac index, as a useful noninvasive predictor of severe PGD development. Finally, we aimed to create a feasible, easy-to-use model to predict severe PGD development using classification and regression tree analysis (CART).

\section{Material and methods \\ Study design}

A prospective cohort study was performed at a single institution (Vall d'Hebron University Hospital, Barcelona, Spain). All lung transplant recipients (LTr) between July 2013 and August 2014 were included in the study. A transthoracic echocardiogram was performed in all patients immediately before entering the operating room. Patients with a poor-quality acquisition of the RVF were excluded from the study. Pulmonary artery catheter data were measured with the Edwards CCOmbo System (Edwards, Irvine, CA, USA) just after placement with the patient sedated and intubated. In addition to echocardiogram or pulmonary artery catheter data, patients' demographic data (sex, age, body mass index and previous lung disease) were recorded. Data regarding pretransplantation nutritional status (protein) and blood volume (haemoglobin), intraoperative characteristics, procedure type, blood product transfusion, inhaled nitric oxide (iNO), cardiopulmonary bypass and lung ischaemia time were also recorded.

The study was approved by the Clinical Research Ethics Committee of Vall d'Hebron University Hospital (PR (AG) 144/2013) and all subjects gave informed consent before inclusion. Donor selection and intraoperative and postoperative management were performed in accordance with the protocols in place at Vall d'Hebron Hospital.

\section{PGD definition}

The primary outcome was appearance of PGD grade 3 (PGD3) at any time point during the first $72 \mathrm{~h}$ postsurgery. The time points for evaluation of PGD3 development were 6, 24, 48 and $72 \mathrm{~h}$ after the start of graft perfusion [2]. In accordance with the report of the International Society for Heart and Lung Transplantation Working Group on Primary Lung Graft Dysfunction [2], PGD3 was defined as the presence of radiographic infiltrates consistent with pulmonary oedema and an arterial oxygen tension $\left(\mathrm{PaO}_{2}\right)$ /inspiratory oxygen fraction $\left(\mathrm{FIO}_{2}\right)$ ratio $<200 \mathrm{mmHg} . \mathrm{PaO}_{2} / \mathrm{FIO}_{2}$ ratio was accurately measured in mechanically ventilated patients. Patients who were not intubated at the time of $\mathrm{PaO}_{2} / \mathrm{FIO}_{2}$ measurement were graded as no PGD3 [2]. In the single-lung transplants, PGD3 development was determined by a 
$\mathrm{PaO}_{2} / \mathrm{FIO}_{2}<200 \mathrm{mmHg}$ and the presence of radiographic infiltrates in the implanted lung [2]. Furthermore, the researchers who graded PGD were blinded to the echocardiographic results and other clinical findings.

\section{Standard echocardiography}

Transthoracic echocardiography was performed in accordance with the current guidelines [21] using a portable echocardiograph (Vivid i; GE Medical Systems, Horten, Norway) equipped with a $2.5-\mathrm{MHz}$ transducer. RVF was evaluated from the modified apical four-chamber view in accordance with current guidelines [18]. Conventional echocardiographic assessment of RVF was performed using four different parameters. First, the RV FAC was calculated as follows:

$$
\mathrm{RV} F A C=\frac{\mathrm{RV} \text { end }- \text { diastolic area }-\mathrm{RV} \text { end }- \text { systolic area }}{\mathrm{RV} \text { end }- \text { diastolic area }} \times 100 \%
$$

Second, the tricuspid annular plane systolic excursion (TAPSE) was calculated as the total excursion of free wall tricuspid annulus from its highest position to its peak descent during ventricular systole by means of the M-mode. Third, Doppler-derived tricuspid lateral annular systolic velocity $\left(S^{\prime}\right)$ was measured by the peak systolic velocity of the lateral corner of the tricuspid annulus. Finally, myocardial performance index (MPI) was measured by the tissue Doppler method in accordance with the current recommendations [19]. From the apical four-chamber view, left ventricular volumes were measured at end-systole and end-diastole, and the left ventricle ejection fraction was calculated using Simpson's biplane model [21]. Left diastolic pattern was estimated using pulsed-wave Doppler recording, from which the $E / A$ ratio was calculated. Finally, in order to estimate right atrial pressure and blood volume status, inferior vena cava collapse was measured from the subcostal window perpendicular to the long axis and proximal to the junction of the hepatic veins [19].

\section{Speckle-tracking echocardiography}

For STE analysis, apical four-chamber view images were obtained using conventional 2D grayscale echocardiography, storing the images at end-expiration and with a stable ECG recording. Care was taken to obtain the best visualisation of the RV, allowing accurate delineation of its endocardial border. Three consecutive heart cycles were recorded and averaged. The frame rate was set between 60 and 80 frames per second. The files recorded were analysed off-line using the commercially available EchoPAC analysis system (EchoPAC version BTO8; GE Vingmed Ultrasound, Waukesha, WI, USA). RV free-wall strain was derived by an accurate manual delineation of the endocardial border of the free wall only, obtaining a region of interest divided into three segments: basal, medial and apical (figure 1). We evaluated only the RV free wall because previous studies have demonstrated a good correlation between free-wall RV 2D strain, RV ejection and RV end-systolic volume measured using cardiac magnetic resonance and right heart catheterisation [19, 22-26]. Longitudinal strain (LS) and strain rate ( $\mathrm{Sr}$ ) data were collected. LS measures the percentage of

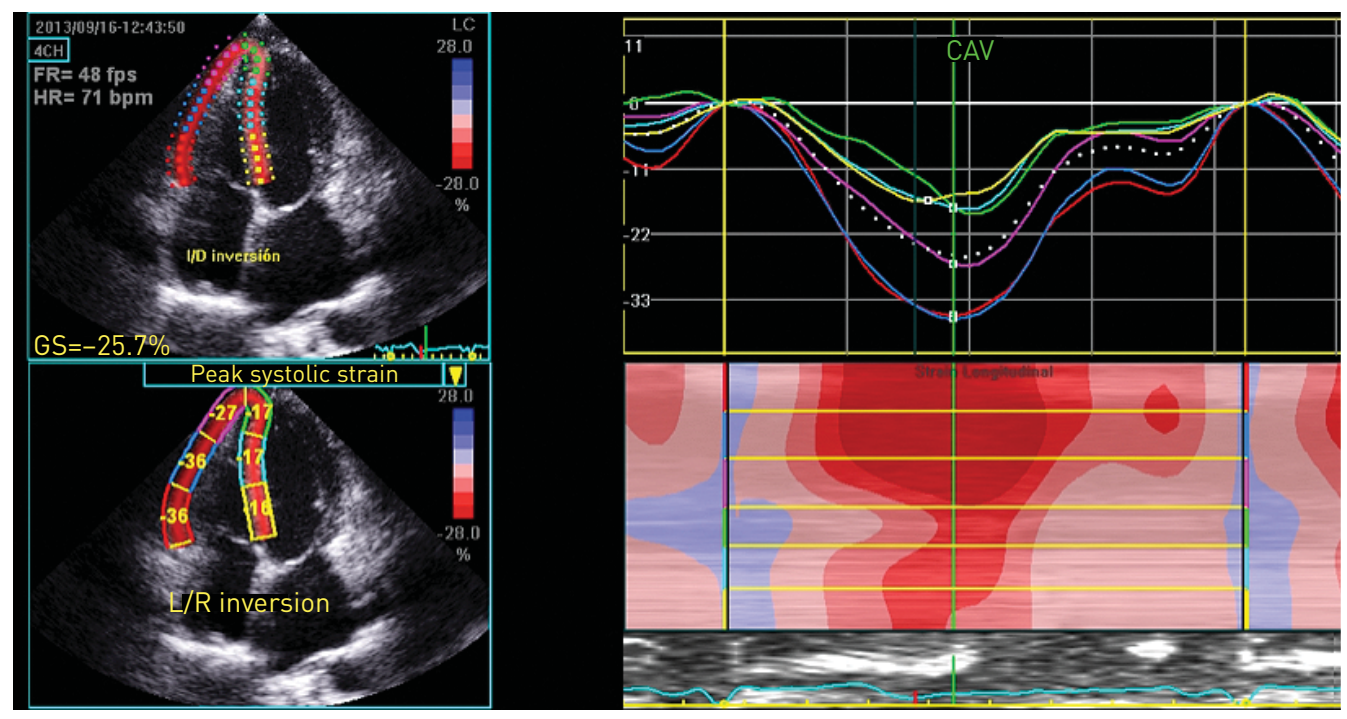

FIGURE 1 Image of right ventricle speckle-tracking strain of a recipient just before lung transplantation. FR: frame rate; fps: frames per second; HR: heart rate; bpm: beats per minute; GS: global strain; L/R: left/right; CAV: closure of aortic valve. 
myocardial deformation; as the RV contracts, the muscle shortens in the longitudinal and circumferential dimensions (negative strain). Sr measures the time course of the deformation.

\section{Statistical analysis}

Baseline characteristics are presented as mean \pm SD for continuous variables and $n$ (\%) for categorical variables. Comparisons between groups were made with the Chi-squared or Fisher's test for categorical variables, and ANOVA or Student t-test for continuous variables, as appropriate. Differences in these variables were assessed according to PGD3 development. Comparisons were considered significant in the presence of a p-value $<0.05$. The Bonferroni method was used to correct multiple comparisons. The sample size calculation was based on data from a retrospective study conducted previously by our group [5]. Accepting an $\alpha$-risk of 0.05 and a $\beta$-risk of 0.2 in a two-sided test and assuming a common standard deviation of 7\%, 31 subjects were necessary in each group in order to recognise a difference in BLS of $5 \%$ or greater as statistically significant.

Logistic regression (LR) analysis was performed to identify predictive models for PGD3 development. Variables with a p-value $\leqslant 0.1$ in the univariate analysis were included in the multivariate analysis. To avoid collinearity, separate models were constructed with either model for invasive cardiac index or BLS. Moreover, to avoid overfitting of the prognostic models, a maximum of one prognostic variable per 10 observed events was allowed for inclusion [27]. In addition, only the variables with statistical significance in the multivariate analysis were included in the model. The ability of the models to discriminate between patients who will have and will not have the event was measured using the area under the receiver operating characteristic curve (AUROC). The Hosmer-Lemeshow test was used to evaluate the goodness of fit of the models. Calibration was measured using Nagelkerke's coefficient of determination and the discrimination of the models was assessed using the AUROC. The two most informative models (one invasive model including cardiac index and another, noninvasive model including BLS) in terms of clinical relevance and accuracy (discrimination and calibration) were selected. The statistical analysis was performed using the Statistical Package for Social Sciences v.18 (SPSS Inc., Chicago, IL, USA).

The prognostic CART analysis was performed on the training set with CART Pro v7.0 software (Salford Systems, San Diego, CA, USA) including, in a one-step fashion, all the variables used in the LR analysis. In brief, the method allows for the construction of inductive decision trees through strict binary splitting, and identifies subgroups of patients who share a specific combination of clinical characteristics and a similar risk [27]. Cut-off points for continuous and ordinal variables were generated automatically by the model based on statistical cost assumptions. The optimal tree was then selected according to its relative misclassification costs, predictive accuracy, and clinical relevance. Receiver operating characteristic curves of classification trees were constructed as described by CоOK and Goldman [28].

To assess the predictive performance of the most informative models obtained by LR and CART, pairwise comparisons of the AUROC were conducted [29].

\section{Results}

$72 \mathrm{LTr}$ were considered for inclusion. Two (3\%) patients were excluded due to poor quality acquisition of the RV. Therefore, 70 LTr were included, of whom 31 (44\%) developed PGD3 at some point during the first $72 \mathrm{~h}$ of the postoperative period, though only $31 \%$ still presented PGD3 at $72 \mathrm{~h}$.

Table 1 shows the preoperative, intraoperative and postoperative characteristics, and outcomes of $\mathrm{LTr}$ included in our study. A higher use of intraoperative iNO was observed in LTr who developed PGD3 (81\% versus $33 \%, \mathrm{p}<0.001)$. However, the increase in nitric oxide administration is due to its use to treat hypoxaemia secondary to early intraoperative PGD3 development ( $8 \%$ versus $64 \%, \mathrm{p}=0.01$ ), without differences in the use of iNO for $\mathrm{PH}(84 \%$ versus $76 \%, \mathrm{p}=0.26)$. These patients also presented nonsignificant trends for female sex $(31 \%$ versus $55 \%, \mathrm{p}=0.05)$, preoperative diagnosis of idiopathic pulmonary fibrosis (IPF) ( $23 \%$ versus $45 \%, \mathrm{p}=0.06)$, use of cardiopulmonary bypass $(\mathrm{CPB})(10 \%$ versus $29 \%, \mathrm{p}=0.06)$ and ischaemic time (299 versus $332 \mathrm{~min}, \mathrm{p}=0.05$ ). All outcomes analysed were worse in patients who developed PGD3.

Just before transplantation, echocardiogram data showed no differences in classical measurements of RVF (TAPSE, FAC, MPI and $S^{\prime}$ ) (table 2). In contrast, higher BLS $(-25.7 \pm 7.3 \%$ versus $-19.5 \pm 6.6 \%, \mathrm{p}<0.01)$, basal $\mathrm{Sr}\left(2.11 \pm 0.59\right.$ versus $\left.1.65 \pm 0.57 \mathrm{~s}^{-1}, \mathrm{p}=0.01\right)$ and medium $\mathrm{Sr}\left(1.35 \pm 0.53\right.$ versus $\left.1.67 \pm 0.46 \mathrm{~s}^{-1}, \mathrm{p}=0.04\right)$ were observed in LTr who developed PGD3. BLS continued to be statistically significant after correction for multiple comparisons (table S1). However, no differences were observed in left ventricular systolic function or preload echocardiographic indicators. Table 3 shows the intraoperative pulmonary artery catheter data; no differences were observed in pulmonary arterial pressure or vascular resistance. However, LTr who developed PGD3 had a higher cardiac index $\left(3.5 \pm 0.8\right.$ versus $\left.2.6 \pm 0.7 \mathrm{~L} \cdot \mathrm{min}^{-1} \cdot \mathrm{m}^{2}, \mathrm{p}<0.01\right)$ and stroke volume index $\left(33 \pm 11\right.$ versus $\left.27 \pm 8 \mathrm{~mL} \cdot \mathrm{m}^{-2}, \mathrm{p}=0.04\right)$ compared with those patients who did not present PGD3. A correlation was found between cardiac index and BLS ( $r=0.45, \mathrm{p}=0.01)$. 
TABLE 1 Lung transplant (LT) recipient characteristics according to primary graft dysfunction grade 3 (PGD3) development in the first $72 \mathrm{~h}$

\begin{tabular}{|c|c|c|c|}
\hline & No PGD3 & PGD3 & p-value \\
\hline LT recipients & 39 & 31 & \\
\hline \multicolumn{4}{|l|}{ Preoperative characteristics } \\
\hline Age years & $55 \pm 12$ & $55 \pm 9$ & 0.84 \\
\hline Females & $12(31 \%)$ & $17(55 \%)$ & 0.05 \\
\hline $\mathrm{BMI} \mathrm{kg} \cdot \mathrm{m}^{-2}$ & $26 \pm 8$ & $27 \pm 5$ & 0.66 \\
\hline Aetiology & & & 0.07 \\
\hline Pulmonary fibrosis & $9(23 \%)$ & $14(45 \%)$ & \\
\hline COPD & $18(46 \%)$ & $14(45 \%)$ & \\
\hline Pulmonary hypertension & $2(5 \%)$ & $2(7 \%)$ & \\
\hline Cystic fibrosis & $6(16 \%)$ & $0(0 \%)$ & \\
\hline Others & $4(10 \%)$ & $1(3 \%)$ & \\
\hline Haemoglobin $\mathrm{g} \cdot \mathrm{dL}^{-1}$ & $14 \pm 2$ & $14 \pm 2$ & 0.88 \\
\hline Plasma protein level $\mathrm{g} \cdot \mathrm{dL}^{-1}$ & $7.4 \pm 0.7$ & $7.3 \pm 0.7$ & 0.44 \\
\hline \multicolumn{4}{|l|}{ Intraoperative characteristics } \\
\hline \multicolumn{4}{|l|}{ Procedure type: } \\
\hline Bilateral LT & $25(64 \%)$ & $20(65 \%)$ & 1.00 \\
\hline CPB use & $4(10 \%)$ & $9(29 \%)$ & 0.06 \\
\hline Blood product transfusion packs & $2(4 \%)$ & $4(6 \%)$ & 0.18 \\
\hline iNO use & $13(33 \%)$ & $25(81 \%)$ & $<0.01$ \\
\hline For hypoxaemia & $1(8 \%)$ & $16(64 \%)$ & 0.01 \\
\hline For $\mathrm{PH}$ & $11(84 \%)$ & $19(76 \%)$ & 0.26 \\
\hline Ischaemia time min & $299 \pm 76.0$ & $332 \pm 64.8$ & 0.05 \\
\hline \multicolumn{4}{|l|}{ Postoperative characteristics } \\
\hline ICU mortality & $0(0 \%)$ & $5(16 \%)$ & 0.02 \\
\hline Hospital mortality & $0(0 \%)$ & $7(23 \%)$ & $<0.01$ \\
\hline 28-day mortality & $0(0 \%)$ & $4(13 \%)$ & 0.03 \\
\hline 90-day mortality & $0(0 \%)$ & $6(19 \%)$ & $<0.01$ \\
\hline ICU LOS days & $22 \pm 23$ & $32 \pm 15$ & 0.04 \\
\hline MV length days & $16 \pm 22$ & $28 \pm 18$ & 0.02 \\
\hline
\end{tabular}

Data are presented as mean \pm SD unless otherwise stated. BMI: body mass index; COPD: chronic obstructive pulmonary disease; CPB: cardiopulmonary bypass; iNO: inhaled nitric oxide; $\mathrm{PH}$ : pulmonary hypertension; ICU: intensive care unit; LOS: length of stay; MV: mechanical ventilation.

The most relevant variables (according to the univariate analysis and our previous knowledge) were chosen for the LR and CART analyses (sex, IPF diagnosis, ischaemia time and CBP use, as well as BLS and cardiac index, iNO for hypoxaemia, and iNO for PH) [4]. The most informative prognostic models obtained by LR are reported in table 4 . The best explanatory model using BLS and the best with cardiac index (models 1 and 2 from table 4) were used to construct several classification trees. Finally, the two trees, one containing cardiac index and the other BLS, that most accurately classified patients according to their risk of developing PGD3 were selected. The first tree (figure 2) allows accurate discrimination of LTr into three subpopulations with different risks of PGD3 development by means of cardiac index (AUROC 0.87): a low-risk group (cardiac index $<2.5$ or $\geqslant 2.5 \mathrm{~L} \cdot \mathrm{min}^{-1} \cdot \mathrm{m}^{-2}$, ischaemia time $\leqslant 288 \mathrm{~min}$ and no IPF) in whom PGD3 risk ranged from $0 \%$ to $18 \%$; an intermediate-risk group (cardiac index $\geqslant 2.5 \mathrm{~L} \cdot \mathrm{min}^{-1} \cdot \mathrm{m}^{-2}$, ischaemia time $<288 \mathrm{~min}$ and IPF) with a PGD3 risk of $64 \%$; and a high-risk group (cardiac index $\geqslant 2.5 \mathrm{~L} \cdot \mathrm{min}^{-1} \cdot \mathrm{m}^{-2}$, ischaemia time $\geqslant 288 \mathrm{~min}$ ) with a PGD3 risk of $86 \%$. This prognostic model had a sensitivity of $84 \%$, a specificity of $82 \%$, and positive and negative predictive values of $86 \%$ and $82 \%$, respectively. The second tree (figure 3) allows the same risk stratification but using a preoperative noninvasive parameter such as BLS; it had a sensitivity of $87 \%$, a specificity of $69 \%$, and positive and negative predictive values of $75 \%$ and $88 \%$ (AUROC 0.81). It identified a low-risk group (BLS $<-23 \%$ and no IPF or BLS $\geqslant-23 \%$ and ischaemia time $<283 \mathrm{~min}$ ), whose PGD3 risk ranged from $11 \%$ to $20 \%$; an intermediate-risk group (BLS $\geqslant-23 \%$ and IPF) with a PGD3 risk of 54\%; and a high-risk group (BLS $\geqslant-23 \%$ and ischaemia time $\geqslant 283$ min) with a risk of PGD3 development of 75\%. AUROCs of the LR and CART models were compared with each other, and the BLS and cardiac index models; no statistically significant differences were found.

\section{Discussion}

This study confirms the hypothesis generated by a previous retrospective study [5] concerning the role of RVF in severe PGD development. It also provides an innovative decision tree based on the use of three 
TABLE 2 Comparison of preoperative echocardiographic variables according to primary graft dysfunction grade 3 (PGD3) development in the first $72 \mathrm{~h}$

\begin{tabular}{lccc} 
& No PGD3 & PGD3 & p-value \\
\hline Lung transplant recipients & 39 & 31 & \\
sPAP mmHg & $51 \pm 27$ & $52 \pm 27$ & 0.88 \\
LVEF \% & $60 \pm 6$ & $59 \pm 5$ & 0.51 \\
E/A & $0.98 \pm 0.32$ & $0.87 \pm 0.26$ & 0.23 \\
IVC collapse \% & $58 \pm 17$ & $62 \pm 15$ & 0.57 \\
Right ventricular function assessment & & & 0.39 \\
FAC \% & $42 \pm 9$ & $44 \pm 10$ & 0.07 \\
TAPSE mm & $19 \pm 4$ & $21 \pm 3$ & 0.16 \\
S $^{\prime}$ cm.s & $12.0 \pm 2.4$ & $13.0 \pm 2.0$ & 0.77 \\
MPI & $0.51 \pm 0.10$ & $0.49 \pm 0.11$ & 0.07 \\
VTI cm & $12.4 \pm 2.4$ & $14.0 \pm 3.1$ & 0.15 \\
LS \% & $-19.1 \pm 5.4$ & $-21.6 \pm 6.1$ & $<0.01$ \\
Free wall & $-19.5 \pm 6.6$ & $-25.7 \pm 7.3$ & 0.14 \\
Basal & $-19.9 \pm 6.6$ & $-23.0 \pm 7.3$ & 1.00 \\
Medium & $-16.9 \pm 5.6$ & $-16.9 \pm 6.5$ & \\
Apical & & & 0.01 \\
Sr s ${ }^{-1}$ & $1.65 \pm 0.57$ & $2.11 \pm 0.59$ & 0.04 \\
Basal & $1.35 \pm 0.53$ & $1.67 \pm 0.46$ & 0.91 \\
Medium & $1.34 \pm 0.51$ & $1.36 \pm 0.51$ & \\
Apical & & & \\
\hline
\end{tabular}

Data are presented as mean \pm SD unless otherwise stated. sPAP: systolic pulmonary arterial pressure; LVEF: left ventricular ejection fraction; IVC: inferior vena cava; FAC: fractional area change; TAPSE: tricuspid annular systolic excursion; $S^{\prime}$ : Doppler-derived tricuspid lateral annular systolic velocity; MPI: myocardial performance index; VTI: velocity time integral; LS: longitudinal strain; Sr: strain rate.

different variables (noninvasive BLS or cardiac index, ischaemia time and IPF) that accurately stratifies LTr according to their risk of PGD3. The decision tree aims to achieve careful perioperative management in order to limit pulmonary overflow.

In agreement with previous reports, our results clearly confirm the high prevalence of severe PGD $[2,3]$ and its significant impact on morbidity and mortality $[6,30]$. Therefore, determining the clinical risk factors associated with PGD is of great clinical interest, since a better identification of those LTr who are at high risk of PGD would allow the implementation of aggressive, early, preventive support. However, despite the significant morbidity and mortality in patients who develop PGD, the associated risk factors remain controversial. A recent meta-analysis showed that female sex, body mass index, preoperative diagnosis of pulmonary fibrosis or primary $\mathrm{PH}$, use of $\mathrm{CPB}$, multiple transfusions and mean pulmonary

TABLE 3 Comparison of intraoperative pulmonary artery catheter parameters according to primary graft dysfunction grade 3 (PGD3) development in the first $72 \mathrm{~h}$

\begin{tabular}{|c|c|c|c|}
\hline & No PGD3 & PGD3 & p-value \\
\hline Lung transplant recipients & 39 & 31 & \\
\hline mPAP mmHg & $30 \pm 12$ & $34 \pm 12$ & 0.14 \\
\hline sPAP mmHg & $41 \pm 22$ & $46 \pm 19$ & 0.30 \\
\hline dPAP mmHg & $23 \pm 7$ & $26 \pm 9$ & 0.17 \\
\hline CPV $\mathrm{mmHg}$ & $12 \pm 5$ & $11 \pm 4$ & 0.58 \\
\hline PVRI dyn $\cdot \mathrm{m}^{2} \cdot \mathrm{cm}^{-5}$ & $314 \pm 116$ & $254 \pm 137$ & 0.23 \\
\hline HR beats per min & $87 \pm 21$ & $91 \pm 13$ & 0.45 \\
\hline Cardiac index $L \cdot \min ^{-1} \cdot \mathrm{m}^{-2}$ & $2.6 \pm 0.7$ & $3.5 \pm 0.8$ & $<0.01$ \\
\hline SVI $\mathrm{mL} \cdot \mathrm{m}^{-2}$ & $27 \pm 8$ & $33 \pm 11$ & 0.04 \\
\hline RVEF \% & $26 \pm 7$ & $30 \pm 9$ & 0.15 \\
\hline
\end{tabular}

Data are presented as mean \pm SD unless otherwise stated. mPAP: mean pulmonary arterial pressure; sPAP: systolic pulmonary arterial pressure; dPAP: diastolic pulmonary arterial pressure; CPV: central venous pressure; PVRI: pulmonary vascular resistance index; HR: heart rate; SVI: systolic volume index; RVEF: right ventricular ejection fraction. 
TABLE 4 Multivariate logistic regression analysis for development of primary graft dysfunction grade 3 development

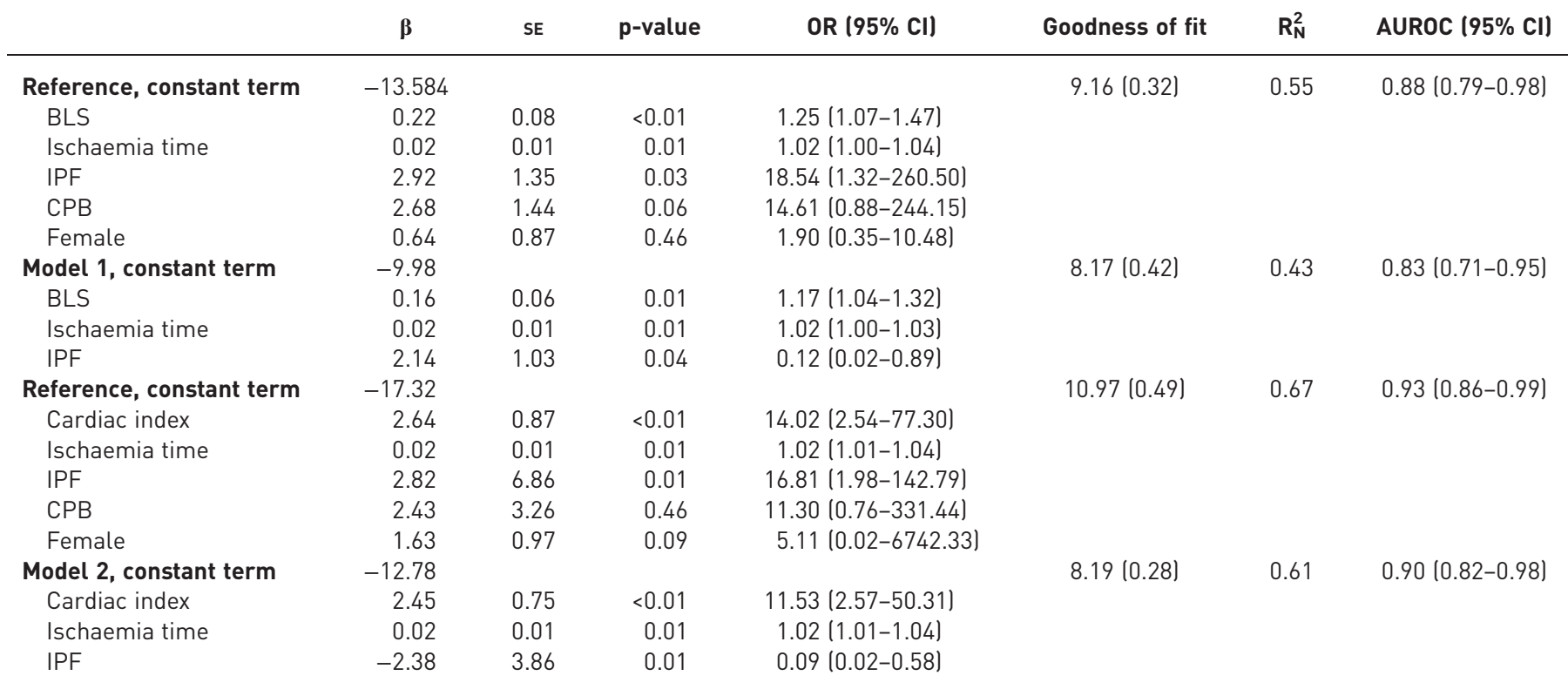

Goodness of fit is presented as the Hosmer-Lemeshow C-statistic (p-value). $\beta$ : regression coefficient; R $R_{N}^{2}$ : Nagelkerke's coefficient of determination; AUROC: area under the receiver operating characteristic curve; BLS: basal longitudinal strain; IPF: idiopathic pulmonary fibrosis; CPB: cardiopulmonary bypass.

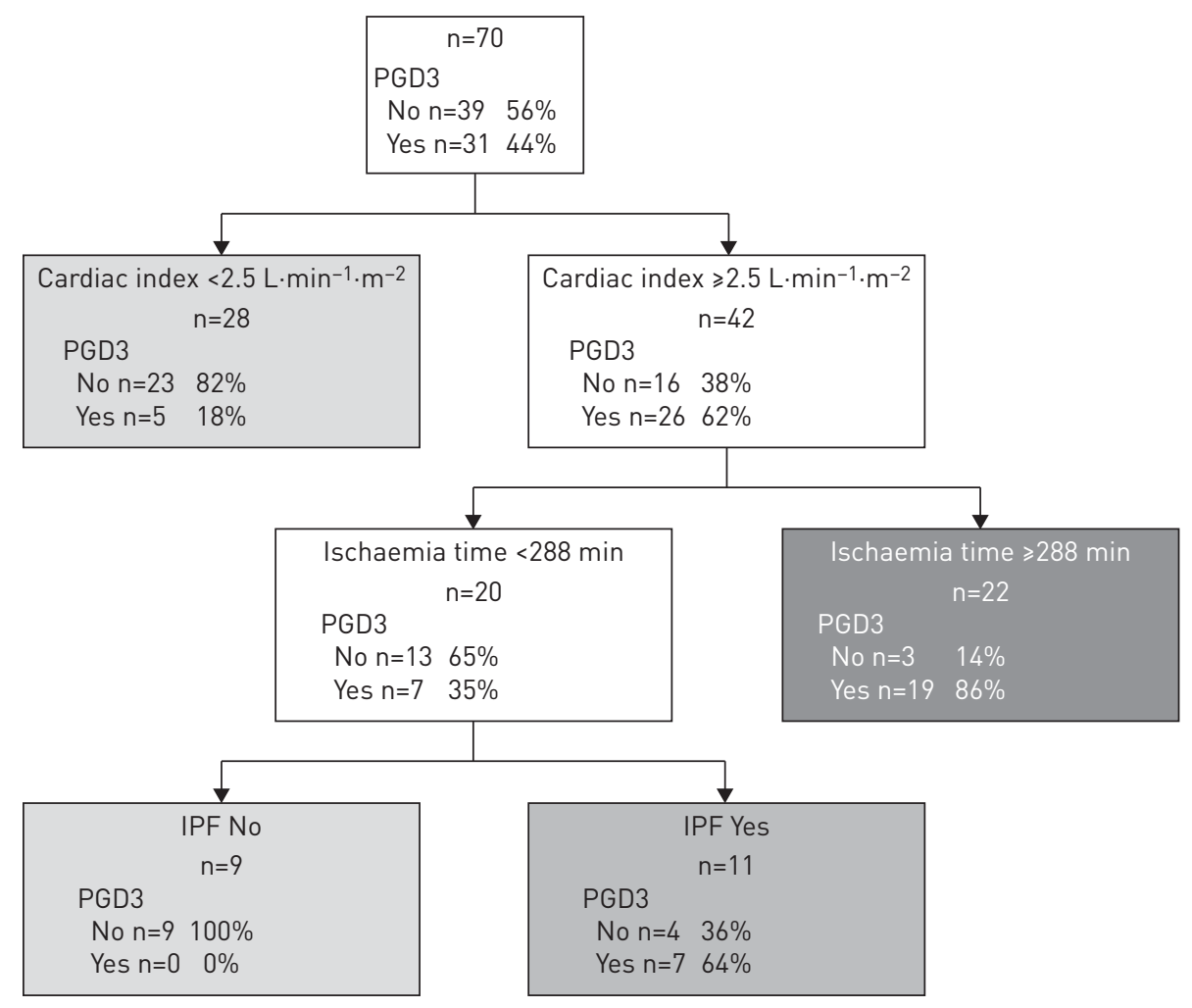

FIGURE 2 Prognostic model for development of primary graft dysfunction grade 3 (PGD3) based on invasive intraoperative cardiac index. IPF: idiopathic pulmonary fibrosis.

arterial pressure (mPAP) were significantly and consistently associated with development of PGD [4]. We also observed that PGD3 patients were more likely to be female, to be diagnosed with IPF, to have longer ischaemia times and to need $\mathrm{CPB}$. Intraoperative use of $\mathrm{iNO}$ was more frequent in patients with PGD: this 


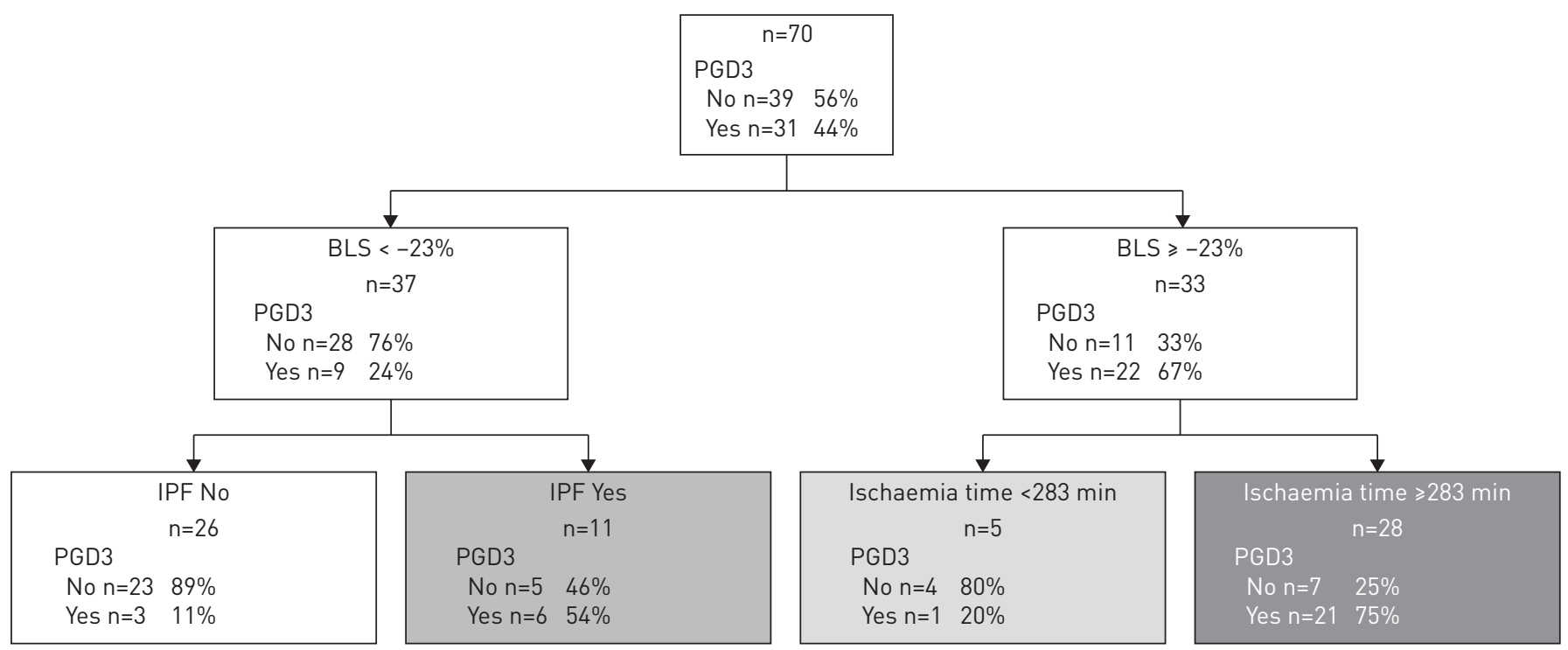

FIGURE 3 Prognostic model for development of primary graft dysfunction grade 3 (PGD3) based on noninvasive preoperative basal longitudinal strain (BLS). IPF: idiopathic pulmonary fibrosis.

should not be considered a risk factor for PGD, but a consequence of the appearance of intraoperative hypoxaemia $[4,16]$. However, we cannot rule out the possible role of iNO in decreasing RV afterload and enhancing RVF. Furthermore, our study failed to show differences in the mPAP.

Preoperative $\mathrm{PH}$ is common among patients with respiratory diseases who are undergoing LT [12]. PH is characterised by chronic RV pressure overload due to pulmonary vascular obstruction. The development of RVF from normal to a compensated (hypertrophied) and then a decompensated state follows the evolution of pulmonary vascular disease [15]. However, after lung implantation in LT, RV afterload is acutely reduced, which increases pulmonary blood flow and the shear stress on the formerly hypoxic pulmonary vascular endothelium. Shear stress leads to capillary leak and impairs graft function [31-37]. The possible role of the RVF in the development of severe PGD was explored by our group in a previous retrospective study that suggested that RVF, measured by BLS, could be an independent risk factor for PGD3 development [5]. Those results were confirmed by the present study using both echocardiography and pulmonary artery catheter data.

Cardiac magnetic resonance is the gold standard for evaluating the RV volume and ejection fraction, but it cannot be applied in all patients. Therefore, patients with $\mathrm{PH}$ are routinely followed using echocardiography, but owing to the complex geometry of the RV, the conventional $2 \mathrm{D}$ echocardiographic assessment of the RVF is considered less accurate $[38,39]$. In addition, the stroke volume of the RV is closely related to the shortening of the longitudinal axis rather than to a reduction in the cavity diameter $[14,24,40]$. In this regard, a novel non-Doppler echocardiographic technology called STE has been proposed as an alternative approach for defining RV longitudinal function, allowing the quantification of global and regional myocardial deformation from standard 2D echo-images without the problem of angle dependence [20, 24, 41-43]. Moreover, STE has the advantage of identifying true contractility rather than tethering or translational motion, which may influence indices like TAPSE or tissue Doppler imaging; it may therefore be a more sensitive parameter for detecting early RV dysfunction and may better predict outcomes [23, 44, 45]. In particular, free-wall RV longitudinal strain has demonstrated higher accuracy and good sensitivity and specificity for predicting depressed RVF [26].

Patients with PH have a lower RV free wall strain than normal controls [24, 43, 46, 47]. However, this involvement is not homogeneous. In $\mathrm{PH}$, there is a pulmonary vascular remodelling with cell proliferation in the pulmonary artery wall $[15,44,48-50]$. Due to the progressive nature of the disease, RV outflow also begins to function as a conduit rather than as a contributor to the overall pump function. This pattern and degree of function disturbances are then compensated for by the mid- and basal RV region, which become the main determinants of the peak ejection timing of the RV [51]. The predominant involvement of the most apical segments was also confirmed in our study, although there was a clear difference in the impairment of the basal segments between patients who developed severe PGD and those who did not.

LTr who developed PGD3 had a higher intraoperative cardiac index. This confirms the hypothesis of our study; that is, that LTr with better RVF have a higher risk of developing severe PGD due to higher 
pulmonary flow. Moreover, BLS is an independent risk factor for PGD3 development. Therefore, by means of a noninvasive examination performed before LT, we were able to predict which patients would have a higher risk of developing severe PGD3. Most importantly, we used two different statistical approaches, LR and CART analysis, for prognostic assessment of the development of severe PGD. Although LR analyses have been widely used, it is sometimes difficult to translate the information in their prognostic models to bedside clinical management. In this regard, CART analyses have been proposed as an interesting alternative. Here, we present a highly intuitive decision tree that, based on the combined use of just three variables (invasive cardiac index or noninvasive BLS, IPF, and ischaemia time), straightforwardly classifies LTr patients into different groups according to their risk of PGD3. It can help to identify patients with a higher risk of developing severe PGD who would benefit the most from an extremely careful perioperative management strategy able to limit pulmonary overflow and, thus, facilitates daily clinical decision-making. On the other hand, the use of BLS allows earlier, noninvasive risk stratification just before LT, without significant differences regarding the use of cardiac index.

The present study has some limitations. The first is that it is a single-centre study with a relatively small sample size. This small size might justify that the trend toward worse left diastolic function in PGD3 patients (lower $E / A$ and higher diastolic pulmonary arterial pressure) could not reach statistically significance. Mostly in the older patient subgroup, this possible diastolic dysfunction combined with increased pulmonary flow could play a role in the development of PGD3. However, this study was specifically designed to determine the effect of RVF on the development of PGD, and the sample size was calculated in accordance with previous data [5]. Consequently, the present study was correctly powered to confirm our hypothesis.

The second limitation is that the transthoracic echocardiography was performed with the patients awake just before surgery, while the pulmonary artery catheter data were collected with the patients sedated and ventilated. In addition, the STE involves manual tracing of the endocardial border, which requires special care and may be particularly difficult in the apical regions of the free wall. The third is that no data about the donor and recipient lung size measurements were provided. However, the ribcage of both the recipient and the donor is meticulously measured. In fact, none of the patients included needed a lung resection due to a discrepancy in lung size. Finally, patients who developed severe PGD had a better RVF, measured by BLS and cardiac index. Although this may not necessarily imply causation, we constructed various LR and CART models using several different variables that have been described as potential risk factors for PGD. Interestingly, BLS and cardiac index have been consistently associated with a higher probability of severe PGD in all models, suggesting that RVF may be considered as an independent risk factor for severe PGD. Nevertheless, despite these potential limitations, this study demonstrates the importance of RVF in the appearance of severe PGD. These results provide new evidence regarding the pathophysiology of PGD, which is the most common early complication of LT, and contributes significantly to patient morbidity and mortality. Thus, understanding the mechanisms of PGD generation will aid the design of therapeutic strategies focused on PGD prevention, which are able to improve outcomes in LTr patients. Another important contribution is the validation of BLS as an independent risk factor for severe PGD development and the proposal of a novel approach, based on CART analysis, which yields an accurate model of great potential clinical value for predicting the risk of severe PGD development.

\section{Conclusions}

Better preoperative RVF is a risk factor for severe PGD development. Therefore, accurate RVF assessment before LT by means of STE is essential to prompt identification of LTr with a higher risk of developing PGD who would benefit the most from an extremely careful perioperative management strategy able to limit pulmonary overflow.

\section{References}

1 Leal S, Sacanell J, Riera J, et al. Early postoperative management of lung transplantation. Minerva Anestesiol 2014; 80: 1234-1245.

2 Christie JD, Van Raemdonck D, de Perrot M, et al. Report of the ISHLT Working Group on Primary Lung Graft Dysfunction part I: introduction and methods. J Heart Lung Transplant 2005; 24: 1451-1453.

3 King RC, Binns OA, Rodriguez F, et al. Reperfusion injury significantly impacts clinical outcome after pulmonary transplantation. Ann Thorac Surg 2000; 69: 1681-1685.

4 Liu Y, Liu Y, Su L, et al. Recipient-related clinical risk factors for primary graft dysfunction after lung transplantation: a systematic review and meta-analysis. PLoS One 2014; 9: e92773.

5 Pérez-Terán $\mathrm{P}$, Roca $\mathrm{O}$, Rodríguez-Palomares J, et al. Influence of right ventricular function on the development of primary graft dysfunction after lung transplantation. J Hear Lung Transplant 2015; 34: 1423-1429.

6 Christie JD, Sager JS, Kimmel SE, et al. Impact of primary graft failure on outcomes following lung transplantation. Chest 2005; 127: 161-165.

7 Christie JD, Kotloff RM, Ahya VN, et al. The effect of primary graft dysfunction on survival after lung transplantation. Am J Respir Crit Care Med 2005; 171: 1312-1316. 
8 Whitson BA, Nath DS, Johnson AC, et al. Risk factors for primary graft dysfunction after lung transplantation J Thorac Cardiovasc Surg 2006; 131: 73-80.

9 Barr ML, Kawut SM, Whelan TP, et al. Report of the ISHLT Working Group on Primary Lung Graft Dysfunction part IV: recipient-related risk factors and markers. J Heart Lung Transplant 2005; 24: 1468-1482.

10 Christie JD, Kotloff RM, Pochettino A, et al. Clinical risk factors for primary graft failure following lung transplantation. Chest 2003; 124: 1232-1241.

11 Fang A, Studer S, Kawut SM, et al. Elevated pulmonary artery pressure is a risk factor for primary graft dysfunction following lung transplantation for idiopathic pulmonary fibrosis. Chest 2011; 139: 782-787.

12 Shorr AF, Wainright JL, Cors CS, et al. Pulmonary hypertension in patients with pulmonary fibrosis awaiting lung transplant. Eur Respir J 2007; 30: 715-721.

13 Aguero J, Ishikawa K, Hadri L, et al. Characterization of right ventricular remodeling and failure in a chronic pulmonary hypertension model. Am J Physiol Heart Circ Physiol 2014; 307: H1204-H1215.

14 Voelkel NF, Quaife RA, Leinwand LA, et al. Right ventricular function and failure: report of a National Heart, Lung, and Blood Institute working group on cellular and molecular mechanisms of right heart failure. Circulation 2006; 114: 1883-1891.

15 Champion HC, Michelakis ED, Hassoun PM Comprehensive invasive and noninvasive approach to the right ventricle-pulmonary circulation unit: state of the art and clinical and research implications. Circulation 2009; 120: 992-1007.

16 De Perrot M, Liu M, Waddell TK, et al. Ischemia-reperfusion-induced lung injury. Am J Respir Crit Care Med 2003; 167: 490-511.

17 Wrobel JP, Thompson BR, Snell GI, et al. Preoperative echocardiographic-defined moderate-severe pulmonary hypertension predicts prolonged duration of mechanical ventilation following lung transplantation for patients with COPD. Lung 2012; 190: 635-643.

18 López-Aguilar J, Piacentini E, Villagrá A, et al. Contributions of vascular flow and pulmonary capillary pressure to ventilator-induced lung injury. Crit Care Med 2006; 34: 1106-1112.

19 Rudski LG, Lai WW, Afilalo J, et al. Guidelines for the echocardiographic assessment of the right heart in adults: a report from the American Society of Echocardiography endorsed by the European Association of Echocardiography, a registered branch of the European Society of Cardiology, and the Canadian Society of Echocardiography. J Am Soc Echocardiogr 2010; 23: 685-713.

20 Mondillo S, Galderisi M, Mele D, et al. Speckle-tracking echocardiography: a new technique for assessing myocardial function. J Ultrasound Med 2011; 30: 71-83.

21 Lang RM, Badano LP, Mor-Avi V, et al. Recommendations for cardiac chamber quantification by echocardiography in adults: an update from the American Society of Echocardiography and the European Association of Cardiovascular Imaging. J Am Soc Echocardiogr 2015; 28: 1-39.

22 Motoji Y, Tanaka H, Fukuda Y, et al. Efficacy of right ventricular free-wall longitudinal speckle-tracking strain for predicting long-term outcome in patients with pulmonary hypertension. Circ J 2013; 77: 756-763.

23 Fukuda Y, Tanaka H, Sugiyama D, et al. Utility of right ventricular free wall speckle-tracking strain for evaluation of right ventricular performance in patients with pulmonary hypertension. J Am Soc Echocardiogr 2011; 24: 1101-1108.

24 Meris A, Faletra F, Conca C, et al. Timing and magnitude of regional right ventricular function: a speckle tracking-derived strain study of normal subjects and patients with right ventricular dysfunction. $J$ Am Soc Echocardiogr 2010; 23: 823-831

25 Filusch A, Mereles D, Gruenig E, et al. Strain and strain rate echocardiography for evaluation of right ventricular dysfunction in patients with idiopathic pulmonary arterial hypertension. Clin Res Cardiol 2010; 99: 491-498.

26 Cameli M, Lisi M, Righini FM, et al. Right ventricular longitudinal strain correlates well with right ventricular stroke work index in patients with advanced heart failure referred for heart transplantation. J Card Fail 2012; 18: 208-215.

27 Augustin S, Muntaner L, Altamirano JT, et al. Predicting early mortality after acute variceal hemorrhage based on classification and regression tree analysis. Clin Gastroenterol Hepatol 2009; 7: 1347-1354.

28 Cook EF, Goldman L. Empiric comparison of multivariate analytic techniques: advantages and disadvantages of recursive partitioning analysis. J Chronic Dis 1984; 37: 721-731.

29 Hanley JA, McNeil BJ. A method of comparing the areas under receiver operating characteristic curves derived from the same cases. Radiology 1983; 148: 839-843.

30 Lee JC, Christie JD, Keshavjee S. Primary graft dysfunction: definition, risk factors, short- and long-term outcomes. Semin Respir Crit Care Med 2010; 31: 161-171.

31 Pierre AF, DeCampos KN, Liu M, et al. Rapid reperfusion causes stress failure in ischemic rat lungs. $J$ Thorac Cardiovasc Surg 1998; 116: 932-942.

32 Halldorsson AO, Kronon MT, Allen BS, et al. Lowering reperfusion pressure reduces the injury after pulmonary ischemia. Ann Thorac Surg 2000; 69: 198-203.

33 Hall SM, Odom N, McGregor CG, et al. Transient ultrastructural injury and repair of pulmonary capillaries in transplanted rat lung: effect of preservation and reperfusion. Am J Respir Cell Mol Biol 1992; 7: 49-57.

34 Hidalgo MA, Shah KA, Fuller BJ, et al. Cold ischemia-induced damage to vascular endothelium results in permeability alterations in transplanted lungs. J Thorac Cardiovasc Surg 1996; 112: 1027-1035.

35 Lu YT, Hellewell PG, Evans TW. Ischemia-reperfusion lung injury: contribution of ischemia, neutrophils, and hydrostatic pressure. Am J Physiol 1997; 273: L46-L54.

36 Pickford MA, Green CJ, Sarathchandra P, et al. Ultrastructural changes in rat lungs after $48 \mathrm{~h}$ cold storage with and without reperfusion. Int J Exp Pathol 1990; 71: 513-528.

37 Mills AN, Hooper TL, Hall SM, et al. Unilateral lung transplantation: ultrastructural studies of ischemia-reperfusion injury and repair in the canine pulmonary vasculature. J Heart Lung Transplant 1992; 1: 58-67.

38 Rajagopal S, Forsha DE, Risum N, et al. Comprehensive assessment of right ventricular function in patients with pulmonary hypertension with global longitudinal peak systolic strain derived from multiple right ventricular views. J Am Soc Echocardiogr 2014; 27: 657-665.

39 Horton KD, Meece RW, Hill JC. Assessment of the right ventricle by echocardiography: a primer for cardiac sonographers. J Am Soc Echocardiogr 2009; 22: 776-792. 
Torrent-Guasp F, Ballester M, Buckberg GD, et al. Spatial orientation of the ventricular muscle band: physiologic contribution and surgical implications. J Thorac Cardiovasc Surg 2001; 122: 389-392.

41 Cameli M, Caputo M, Mondillo S, et al. Feasibility and reference values of left atrial longitudinal strain imaging by two-dimensional speckle tracking. Cardiovasc Ultrasound 2009; 7: 6.

42 Wakami K, Ohte N, Asada K, et al. Correlation between left ventricular end-diastolic pressure and peak left atrial wall strain during left ventricular systole. J Am Soc Echocardiogr 2009; 22: 847-851.

43 Li Y, Xie M, Wang X, et al. Right ventricular regional and global systolic function is diminished in patients with pulmonary arterial hypertension: a 2-dimensional ultrasound speckle tracking echocardiography study. Int $J$ Cardiovasc Imaging 2013; 29: 545-551.

44 Okumura K, Humpl T, Dragulescu A, et al. Longitudinal assessment of right ventricular myocardial strain in relation to transplant-free survival in children with idiopathic pulmonary hypertension. J Am Soc Echocardiogr 2014; 27: 1344-1351.

45 Park J-H, Negishi K, Kwon DH, et al. Validation of global longitudinal strain and strain rate as reliable markers of right ventricular dysfunction: comparison with cardiac magnetic resonance and outcome. J Cardiovasc Ultrasound 2014; 22: 113-120.

46 Pirat B, McCulloch ML, Zoghbi WA. Evaluation of global and regional right ventricular systolic function in patients with pulmonary hypertension using a novel speckle tracking method. Am J Cardiol 2006; 98: 699-704.

47 López-Candales A, Dohi K, Bazaz R, et al. Relation of right ventricular free wall mechanical delay to right ventricular dysfunction as determined by tissue Doppler imaging. Am J Cardiol 2005; 96: 602-606.

48 Dambrauskaite V, Delcroix M, Claus P, et al. Regional right ventricular dysfunction in chronic pulmonary hypertension. J Am Soc Echocardiogr 2007; 20: 1172-1180.

49 Naito $\mathrm{H}$, Arisawa J, Harada $\mathrm{K}$, et al. Assessment of right ventricular regional contraction and comparison with the left ventricle in normal humans: a cine magnetic resonance study with presaturation myocardial tagging. $\mathrm{Br} \mathrm{Heart}$ J 1995; 74: 186-191.

50 Fayad ZA, Ferrari VA, Kraitchman DL, et al. Right ventricular regional function using MR tagging: normals versus chronic pulmonary hypertension. Magn Reson Med 1998; 39: 116-123.

51 Calcutteea A, Lindqvist P, Soderberg S, et al. Global and regional right ventricular dysfunction in pulmonary hypertension. Echocardiography 2014; 31: 164-171. 